Article

\title{
A Paradigm Shift in Biology?
}

\section{Gennaro Auletta}

Pontifical Gregorian University, Piazza della Pilotta, 400187 Rome, Italy;

E-Mail: gennaro.auletta@gmail.com

Received: 16 July 2010 / Accepted: 6 September 2010 / Published: 13 September 2010

\begin{abstract}
All new developments in biology deal with the issue of the complexity of organisms, often pointing out the necessity to update our current understanding. However, it is impossible to think about a change of paradigm in biology without introducing new explanatory mechanisms. I shall introduce the mechanisms of teleonomy and teleology as viable explanatory tools. Teleonomy is the ability of organisms to build themselves through internal forces and processes (in the expression of the genetic program) and not external ones, implying a freedom relative to the exterior; however, the organism is able to integrate internal and external constraints in a process of co-adaptation. Teleology is that mechanism through which an organism exercises an informational control on another system in order to establish an equivalence class and select some specific information for its metabolic needs. Finally, I shall examine some interesting processes in phylogeny, ontogeny, and epigeny in which these two mechanisms are involved.
\end{abstract}

Keywords: information; teleology; teleonomy; epigeny; complexity; function

\section{Introduction}

There is a large consensus in the scientific community that molecular biology has brought a terrific quantity of important results in the last few decades. The question naturally arises whether this approach will be also so fertile in the future, in particular across this century. As a matter of fact, the GENOME Project [1] has brought very relevant results but also has shown the necessity to step further, in particular towards the necessity to take into account a sort of proteinomics. Some new developments question how fruitful the traditional approach will still be in the long run if taken as the sole available methodology. Only to mention some of these, we can think about 
- The growing importance of epigenetic gene-control networks, where the stress is on branching events in development, that is, on binary choices that determine the subsequent developmental course, instead of a linear determination of the phenotype by the genome [2-5].

- Neglecting the relevance of introns seems growingly difficult to maintain. To give an example, the small nucleolar RNAs (snoRNAs) are encoded in the introns of other genes and serve to RNA maturation. This shows the complexity of control mechanisms in the cell. This complexity can be seen also in RNA or protein decay, as also textbooks acknowledge today [6].

- The difficulty to explain with traditional methods certain findings: For instance, the yeast $\alpha 2$ protein and the Drosophila's engrailed protein have almost the same structure and functionality but share only 17 amino acid residues over 60 [7]. It is to consider that the shared amino acid residues do not constitute the active domains of the proteins.

- Recently, it has been proposed to search the onset of cancer in a global disfunction of the epigenetic network and especially in cell-cell signaling [8].

- The extensively acknowledged relevance of top-down processes in neurosciences. This is remarkably evident in the endogenous production of dopamine in the brain of rats exploring their maze [9].

The growing perception is that the traditional conceptual framework of biology, which has been drawn from classical mechanics, is no longer adequate. According to this traditional approach, any biological functionality can be explained in pure chemical or mechanical terms. Are the above examples hinting at the necessity of widening the domain of the currently acceptable scientific explanations (thus likely suggesting the existence of fundamental biological mechanisms so far overlooked)? Or are they merely puzzles to be possibly solved within the present scenario? Of course, it is very difficult to judge right now, and future achievements will help to settle the issue. I recall that the theory of self-organization and of complex systems was perceived by many as contributing to such a paradigm shift [10] a couple of decades ago. But this has not actually been the case, since no new explanatory mechanism has been so far provided but most of the work in this field is descriptive. The mentioned recent developments raise again the problem.

If we are in the mid of a paradigm shift, we need something more than some make-up. Obviously, we shall not abandon traditional molecular and reductionist methodology, because of its crucial importance for biology. However, the latter approach would be necessarily inserted in a new conceptual framework, since if we are really faced with an imminent turning point, we need new conceptual tools that are able to also account for already known phenomena. In the following, I shall show that the language of physics cannot remain centered on notions like mass, energy, speed of reaction, etc., but should be expressed with the help of concepts like constraints, degenerate states and processes, differential timing and irreversible dissipative events, dynamical itinerancy, information sharing and selecting, equivalence classes, i.e., in a non-strictly mechanistic conceptual framework $[11,12]$. This would foster the acceptance of those new dimensions of biological explanations that the examples provided seem to call for.

After having briefly stated the main aspects of the concept of information in biology and its relative independence from chemical activity [Section 2], I shall introduce the key notion of complexity 
[Section 3]. In Section 4 the first relevant mechanism is introduced: Teleonomy. What I have previously analyzed in mathematical terms is here treated in a qualitative way. An analysis of the selection system of the organism follows [Section 5]. The second key mechanism, namely teleology, is introduced in Section 6. In the examples given here we have again a qualitative treatment of what is dealt with in more formal terms than in the previous section. Finally, in Section 7, some conclusive remarks follow.

\section{Information and Chemistry}

An indispensable step to properly understand the issues of some specific biological mechanisms, which constitute the bulk of this paper, is to clearly assess the truly informational status of DNA, its main characters and consequences. DNA satisfies all the requirements of information codification:

(1) The existence of a finite set of mutually exclusive elementary units (A, T, C, G) that

(2) Can be linearly combined (giving rise to a sequence).

(3) Every combination must give rise to some "words" (codons) following certain rules: The codons are triplets, there are start and stop signals, AG and CT are subcodes allowing creation of templates.

Figure 1. The DNA sequence AAT. Note that the bases remain here essentially untouched and chemical connections only concern the sugar-phosphate backbone. Therefore, base pairs maintain essentially the same structure in any sequence. It is precisely this property allowing DNA to be a good storage of information.

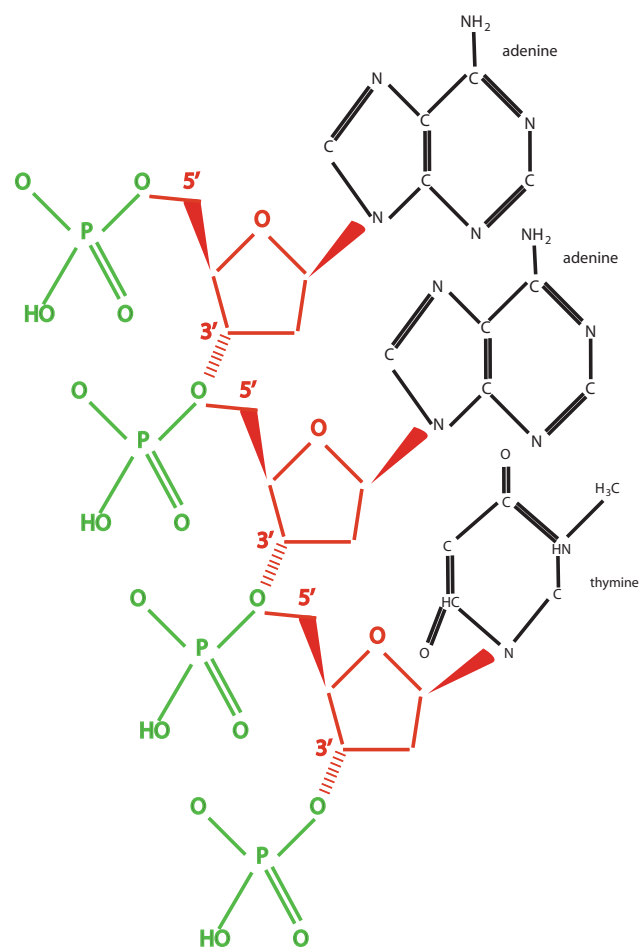

(4) There exist also other elementary units and relative translation rules: The two mentioned subcodes and the RNA code ( $U$ instead of $\mathrm{T}$ ). This suggests a former phase of life with a larger variety of codes, from which, through fine-tuning due to selective pressure, the actual code has been born. 
This is also shown by the possibility to artificially produce new base pairs: The so-called bases $\mathrm{S}$ and $\mathrm{Y}$ have been added to the four traditional ones, by producing a sort of six bases code allowing the production of $6^{3}=216$ different amino acids [13].

It is possible to consider information coding in DNA as a metaphoric way of speaking [14], but I disagree. Indeed, let us suppose that certain configurations of DNA molecules were privileged due to the fact that the bindings of their bases were much stronger than they would be for any other distribution of bases; then such a DNA would have no information content [15]. This is actually the case for atoms and ordinary chemical molecules: Since the orderly structure is here due to a maximum of stability, corresponding to a minimum of potential energy, the orderliness of such molecules lacks the capacity to function as a code. The pattern of atoms forming a crystal is another instance of order without appreciable information content. Therefore, whatever may be the origin of a DNA configuration, it can function as a code only if its order is not a deterministic consequence of the forces due to potential energy. The issue of a certain order or configuration of elements (bases) must be as physically indeterminate as the sequence of words is on a printed page: As the meaningful arrangement of words on a printed page is extraneous to the physics and chemistry of the printed page, so is the base sequence in a DNA molecule as extraneous to the chemical forces at work in the DNA molecule. Information is indeed a formal quantity. The possibility to combine DNA "letters" in this way is guaranteed by the fact that base pairs enter in sequences without changing their shape [Figure 1]: To obtain information codification, it is necessary that chemical bonding and information combinatorics be kept separated (the bases only pair across the two strands and not along the same strand). In other words, the sequence of bases cannot depend on chemistry and indeed does not. This result can be obtained only with large (complex) molecules (which have significant mutual information among the components and allow also for the possibility of local information codification), a part of which permits chemical bonds (the sugar-phosphate backbone) while the informational units will be kept separated (the DNA bases).

This is however not sufficient. The true novelty with the genetic code relative to any other prebiotic chemical is that it is both complex (in their constituents and chemical constraints) and simple (linearly, informationally ordered): It is precisely for this reason that it can give rise to a set of instructions incorporated in the mRNA. Indeed, to have instructions, we need a further requirement relative to information codification: The arrangement of the words (the triplets) cannot be random: Technically speaking, here we need a permutation (where the order of the elements is meaningful) and not a simple combinatorics (where the order of the elements does not matter). This is necessary, since otherwise the sequence of DNA could not be read univocally. Indeed, it is well known that the letters A, C, U can give rise to histidine (in the arrangement CAU), leucine (CUA), isoleucine (AUC), serine (UCA), threonine (ACU), or tyrosine (UAC). I also remark that no single amino acid can be coded by the same basic elements differently permuted, thus avoiding ambiguities. To accomplish all that, however, the separation between chemical bonds and information combinatorics is not the only requirement. Indeed, chemical constraints are necessary to pick out only certain reactions and combinations out of an initial larger variety. It is a selection and amplification process. Those bases would be finally preferred that allowed self-replication with highest fidelity. These initial constraints can be largely found in the chemistry of the protein. Apart from redundancy (i.e., the fact that the same protein can be coded through different codon sequences), amino acids show important correlations concerning [16]: 
(a) Hydrophobicity,

(b) Energy dependence with respect to volume, and

(c) Correlation between these first two correlations.

These physico-chemical properties are those enabling the genetic code: Only amino acids satisfying the requirements expressed by the first two correlations should enter into the genetic code table, in which their hydrophobicty and a volume parameter determine their respective positions in the code.

A very important aspect is the following: The DNA is chemically inert and therefore (1) It must be unpacked and chromatin be unmethylated, (2) it must be expressed through the action of complex proteins, (3) it must be translated in a set of instructions (from which eukaryotic introns have been removed). This strongly contributes to the control on the mechanisms giving rise to new proteins and therefore to the faithfulness of both transcription and translation. Indeed, in a very early stage of life probably dominated by RNA [17], the risk of interferences [18] was very high, precisely because RNA is always active. This shows that codified information is not necessarily read and even should not when it is not necessary for the operation at hand. Indeed, production of specific proteins must be continuously kept under control and damped or enhanced depending on the situation. A nice example is represented by chemotaxis, as we shall see below. This suggests that we must sharply distinguish between an inactive (or dormant) state of information, when it is purely codified information, and an active state, when it is transcribed in a set of instructions represented by the mRNA. It would be a considerable error to deny the status of information to DNA only because it is inactive. This would mean assuming that information is only what is actually communicated, probably as a consequence of a distortion of the classical theory of information [19], a distortion which neither takes into consideration, nor accounts for, the full range of information in our physical world [20,21].

\section{Complexity}

Organisms are complex systems [22,23]. Complex systems are a variety of self-organizing systems. They show very interesting properties that we shall explore step by step. The most important one is represented by the fact that they are hierarchical systems. This is evident in the organization of DNA, showing different levels of order: DNA is packed into nucleosomes, which consists of two full turns of DNA wound around an octameric histone core (histones act as spools around which DNA winds and they play a role in gene regulation) plus the adjacent linker DNA.

Self-organization only means the constitution of a pattern. With pattern, I understand a relationship among elements in a set such that, when an arrangement of a subset of these elements is specified, the probability to guess the arrangement of the remainder generally increases with the size of the previous subset. Patterns are therefore "flat". They are non-linear mathematical structures created when there are both positive (amplifications of signals) and negative (differential damping of signals) feedback. An easy example is represented by the so-called baker's map [24].

Complex systems, instead, present several levels each of which can instantiate one or more patterns. Living complex systems show a specificity relative to any abiotic self-organizing system: The latter kind of self-organizing systems like Bénard cells are dependent on entropic fluxes that are not controlled by 
themselves, and this is the main relation they have with the environment [Figure 2]. These fluxes are also the core of the organism's metabolic system, but here, different from the case of abiotic physical systems, such entropic fluxes are controlled from the inside, that is, by the organism itself.

Figure 2. If the upper plate is heated slowly, the heat will pass from the upper plate to the liquid and will be transferred through the liquid to its bottom layer by thermal conduction. In thermal conduction there is no bulk motion of the liquid but rather a greater thermal motion of the molecules that causes the transfer of heat from the warmer layers to adjacent cooler layers. However, as the temperature of the upper layer is increased, a stage is reached (critical temperature) where the liquid overcomes its viscosity (the internal friction which opposes movement) and begins to undergo bulk motion. This results in a transport of heat by convection currents. The currents are not random but rather they lead to the formation of patterns, and often one first sees small convection cells called Bénard cells. Each cell runs either clockwise or counterclockwise (this depends on the long-ranging correlations).



Let us now introduce some mathematical considerations about complexity. The total entropy of the systems $a$ and $b$ (these could be two different strings of symbols) is given by

$$
H(a, b)=H(a)+H(b)-I(a: b)
$$

where

$$
H(a)=-\sum_{j} p(j) \lg p(j) \quad \text { and } H(b)=-\sum_{j^{\prime}} p\left(j^{\prime}\right) \lg p\left(j^{\prime}\right)
$$

$j$ 's and $j^{\prime}$ 's being different sets of code elements or of elementary events not necessarily of the same number, and $\sum p(j)=\sum p\left(j^{\prime}\right)=1$. The term $I(a: b)$ is the mutual (shared) information between systems $a$ and $b$ and it expressed the amount of order of the compound system. A way to express this quantity is

$$
\begin{aligned}
I(a: b) & =H(a)-H(a \mid b) \\
& =H(b)-H(b \mid a)
\end{aligned}
$$

where $H(a \mid b)$ and $H(b \mid a)$ are the conditional entropies of $a$ on $b$ and of $b$ on $a$, respectively [Figure 3]. They express how much the entropy (the amount of disorder) of a system does not depend on the entropy of the other system. This allows us to express the total entropy $H(a, b)$ of the compound system as a sum of an ordered (the mutual information) and a disordered (the conditional entropies) part:

$$
H(a, b)=I(a: b)+H(a \mid b)+H(b \mid a)
$$


Mutual information only expresses order. However, when dealing with complexity we need something more. We must find a measure of complexity that puts together two different aspects:

Figure 3. Graphic representation of mutual information: It is easy to verify that $I(a: b)=$ $H(a)-H(a \mid b)$, where $H(a)$ is the whole set on the left (blue and red regions), $H(a \mid b)$ is the blue region, $I(a: b)$ the red region.

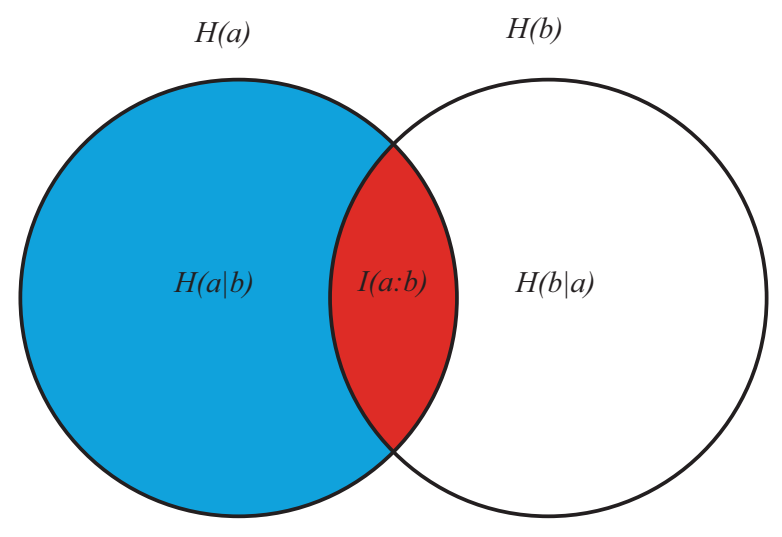

(1) The differentiation of the system in relative independent parts (in some cases, information encapsulation between different levels, like the different levels of DNA packaging from the double strand to the chromosome, and modularity at the same level, like the different segments of DNA exons separated by introns) and

(2) A global order (coherence of the whole).

Let us consider also an external environment with entropy $H(e)$, whose information our strings will store. According to Equation (3), we can write

Figure 4. Complexity of the system $a+b+e$ : It is the whole colored region minus the red region [Equation (11)].

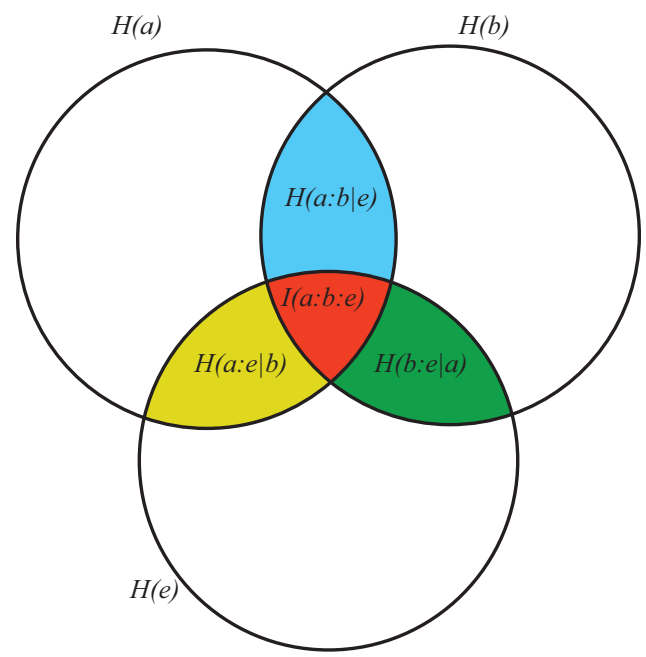

$$
H(a \mid e)=H(a)-I(a: e) \quad \text { and } \quad H(b \mid e)=H(b)-I(b: e) .
$$


Analogously, we can write a conditional entropy of both the systems $a$ and $b$ on $e$, that is,

$$
\begin{aligned}
H(a, b \mid e) & =H(a, b)-I(a, b: e) \\
& =H(a \mid e)+H(b \mid e)-H(a: b \mid e)
\end{aligned}
$$

where an inspection of Figure 4 can be very useful. The quantity $I(a, b: e)$ represents the mutual information between $a$ and $b$ on the one hand and $e$ on the other; it is given by:

$$
I(a, b: e)=I(a: e)+I(b: e)-I(a: e: b)
$$

where $I(a: e: b)$ (the red region in Figure 4), in analogy with Equation (3), is defined by

$$
I(a: e: b)=I(a: e)-H(a: e \mid b)
$$

and represents the overall information shared by $a, b$, and $e$. Note that the quantity $I(a: e: b)$ is symmetric, as expected by a true mutual information [Equation (3)]. Indeed, we also have

$$
\begin{aligned}
I(a: e: b) & =I(a: e)-H(a: e \mid b) \\
& =I(b: e)-H(b: e \mid a)
\end{aligned}
$$

We can use the previous formalism for defining the complexity of the system $a+b+e$ when informational aspects are involved as [Figure 4]

$$
\begin{aligned}
& C(a, b, e)=[I(a: b)-I(a: b: e)] \\
& \quad+[I(a: e)-I(a: b: e)]+[I(b: e)-I(a: b: e)] \\
& =H(a: e \mid b)+H(b: e \mid a)+H(a: b \mid e)
\end{aligned}
$$

It is interesting to note that the difference between the maximal entropy attainable by a system and the entropy of its current state is precisely due to its structural order. Indeed, by considering again Figure 4, it becomes immediately evident that by increasing the colored region, the whole surface given by the combination of the three circles takes down.

Figure 5. The powerfulness of the formalism shown in Equation (11): Any of the mixed terms on the last line can be considered a combination of order and disorder. If we take the term $H(a: e \mid b)$, in particular, (a) shows that it an be interpreted as $I(a: H(e \mid b))$ while (b) shows that it can be equivalently interpreted as $H(I(a: e) \mid b)$.
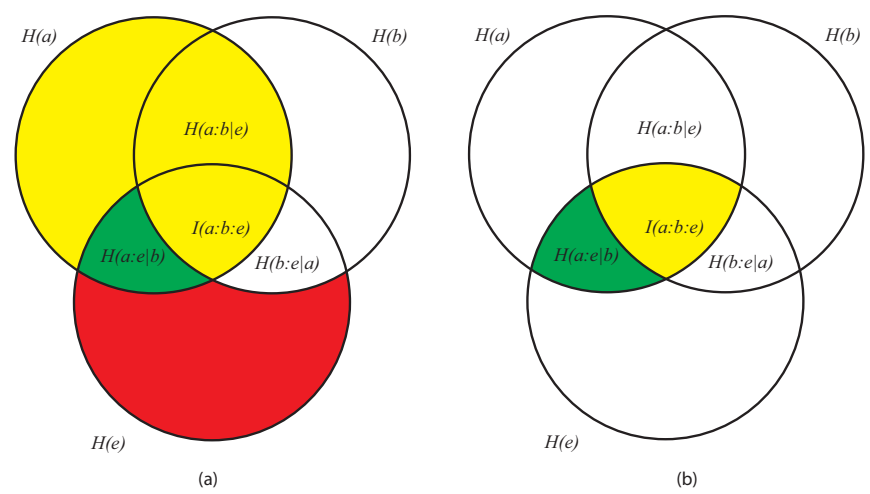
Here, I wish to stress that patterns can be combined to give rise to other patterns, and complex systems can give rise to other complex systems. This is evident when several organism give rise to a whole ecosystem. Now, the crucial point is that mutual information, the quantity occurring several times in first line of Equation (11), does not require that all the involved systems display codified information but only some combinatorics. Indeed, it has to do with the interdependencies among systems and their components. This allows that, together with thermodynamic interdependencies, complex informational-entropic dependencies are established that can then result in new, unexpected informational features. As a matter of fact, only conditional entropies of special kind are present in the last line of Equation (11). They are of special kind, thus allow for new forms of information codification. Indeed, the case of organisms is such that there is always information nested somewhere. To understand this crucial point, let us have a closer look at the expressions of the form $H(a: e \mid b)$ occurring in the last line of Equation (11). These quantities can be equivalently considered in two alternative ways:

- As the conditional entropy between the information shared by $a$ and e, i.e., $I(a: e)$, on the one hand, and a third system $b$, on the other, that is, as $H(I(a: e) \mid b)$, or also

- As the information shared by $a$, on the one hand, and the conditional entropy between $e$ and $b$, on the other $(H(e \mid b))$, that is, as $I(a: H(e \mid b))$.

The relevance of this equivalence and of the relative expressions is that the latter show both conditional entropy and order as expressed by mutual information, and this is in accordance with an intuitive understanding of complexity as a mix of order and disorder.

There are therefore several advantages of the measure (11) of complexity:

(1) It displays both coherence (order: expressed by the dependencies of the subsystems $a, b$, and $e$ ) and autonomy of the parts (expressed by the lack of the mutual information $I(a: b: c)$ ). This means that growing of complexity is not proportional to growing of global order, as already anticipated.

(2) At a global level, it does not directly contain informational terms but only conditional-entropic ones. This means that complexity shows global patterns and functions that are independent of any explicit information coding, even if stemming from information codification.

(3) At a local level (the pairwise relations between the three subsystems) it does not require everywhere, but allows for possible information exchange, sharing, and codification.

When the number of systems grows there are much more possibilities of partial interdependencies, which is the real quintessence of complexity.

\section{Teleonomy}

\subsection{Mechanical and Teleonomic Explanations}

In the following I shall make use of the terminology introduced by J. Monod [25], according to whom teleonomy is the basic property of living beings to be objects endowed with a project, represented in their structures and realized through their performances. Moreover, he stressed that such a property 
denotes the ability of organisms to build themselves through internal forces and mechanisms (in the expression of the genetic program) and not external ones, implying a freedom relative to the exterior. Therefore, teleonomy amounts to the quantity of information that must be transmitted invariantly over the generations in order that the specific organism survives as a biological species. It is important to recall that Monod clarified that teleonomy does not imply to have goals, i.e., a teleology. He even rejected this notion. I agree with him about the fact teleonomy does not show goals, but I shall use the quite different notion of teleology later on. I believe that the reason of Monod's rejection of the notion of teleology is due to the fact that he did not consider the ontogenetic activity of the individual organism (like in chemotaxis) but took especially into account phylogeny and some very basic epigenetic processes. This was certainly due to the progress of biology at that times; to date, I would rather make use of this notion in a specific way, as we shall see below.

Additionally, organisms have a relation with the environment that is also informational. In particular, they are able to use environmental signals and stimuli that are in principle a source of disturbance and even noxious to their needs. Indeed, any variation in the environment is immediately a threat for the organism's homeostasis (it can eventually become a new source of stability only thank to an appropriate reaction). Before discussing this aspect, let me stress the way in which the information contained in the DNA is related with the phenotype. As a matter of fact, the information contained in a genome alone does not constitute the whole information necessary to build the corresponding phenotype. And this holds true for the information contained in the embryo of a multicellular organism. Any organism (and especially a multicellular organism) also depends on environmental cues and feedback circuits to mature. And at any stage of the development an organism shows a quantity of structural information-for instance, the body plan-bigger than that contained in its genome or its embryo [26].

Biological systems are able to integrate complexity and information in a peculiar way: They integrate environmental cues in their own developmental path and therefore put them — to a certain extent—at their service (similar consideration are also true for the internal environment during the different operation of the cells we have introduced before) [27]. Such processes are common also in adult phenotype. An elementary example is also provided by protein folding [28]. The reason for this is that the phenotype or the proteins are a black box relative to the genome [Figure 6]. We see that there is no Lamarckian instructionalism even inside a single organism. The genome starts an informational process that leads to a stable final state (the mature form or the three-dimensional configuration of the protein) only by integrating environmental signals that are generated independently of the organism or by integrating cues that, for instance, come from the metabolic system and are also independent of the specific protein considered. However, the organism is also able to partially canalize the action of the external or internal environment producing a good fit (appropriate to the current operation). It is a phenomenon of co-adaptation [29,30], a higher manifestation of self-organization. 
Figure 6. A good example of teleonomic process: Development (I consider here only the aspects relevant for the issue of this section and not other ones that also play a major role). The immature phenotypic system from which the mature form is developed is a black box to the genetic system (as well as to the environment). Notwithstanding, certain informational processes coming from the DNA have been integrated in a feedback circle with independent environmental signals in order to give rise to a stable mature configuration. These two heterogeneous types of inputs have been selected together at a phylogenetic level (co-adaptation). In other words, the genetic and environmental systems are both independent in their modality of action and tuned one another due to an effect of natural selection. In this way, the final result is not simply the sum of these two independent modes of action. The anti-feedback is the way in which the organism resists to environmental pressures (negative feedback) and is able to tune the external environment to its needs trying to restore its own homeostasis.

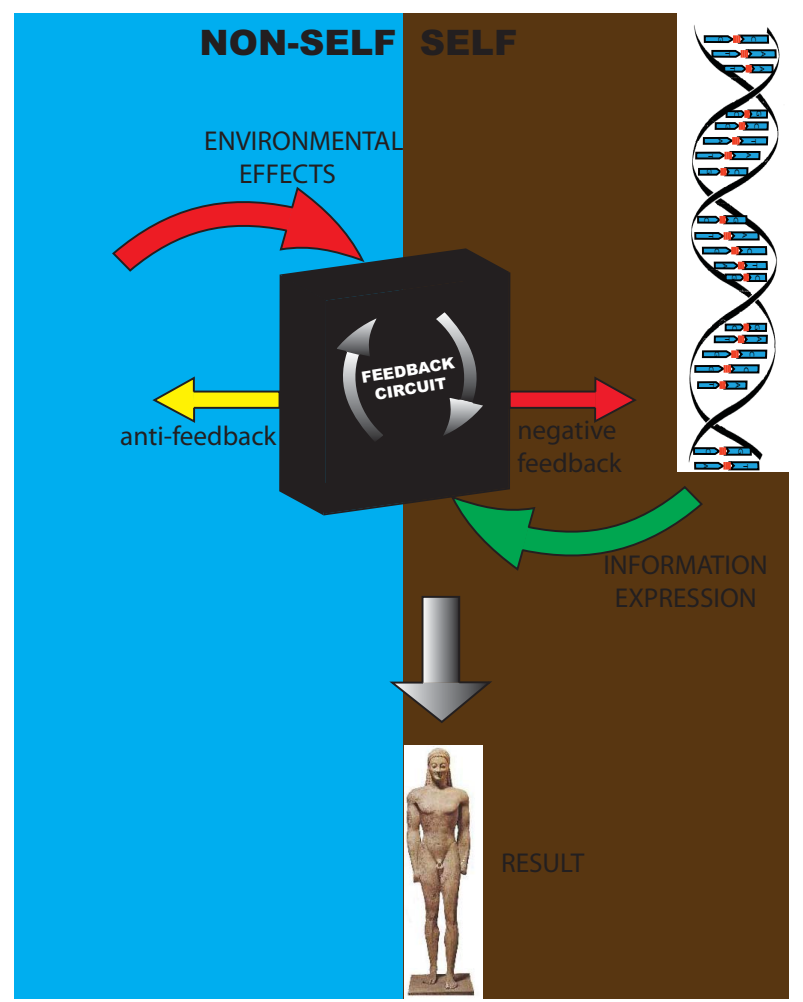

This co-adaptation is also the reason why, from another point of view, the information contained in the genome is overabundant, in the sense that it is never fully expressed: For instance, only a subset of the possible proteins, potentially codified by the genome, is built inside a single cell or, in the case of multicellular organisms, any cell—during development—becomes specialized by expressing (selecting) a subensemble of the information it potentially contains. It is precisely the difference between memory or program and current state to give to biological systems their distinctive temporality. With temporality I understand a rupture of time isotropy, such that

- Future and past states cannot be deduced from the current state in which the system is.

- There is irreversibility, a feature that is strictly connected with the complexity of biological systems. 
A. Lotka was one of the first scholars to understand that even evolution of life is an irreversible process [31]. According to him, evolution is indeed characterized by lags and leads that determine irreversibility, since in the case of lags, the actual and future state of the organism will depend on its previous history.

A scientific theory cannot simply provide a description of its objects but must necessarily provide explanatory mechanisms. Traditional molecular-biology (and in general classical-mechanical) explanations rely on mechanical causation. In mechanical causation, once the causes are given, the effects are certainly produced, so that any classical causal path is univocal. Moreover, any mechanical explanation is in principle about single systems, properties, behaviors. When in molecular biology or in classical mechanics statistical methods are used, it is so only because it is practically impossible to follow single trajectories or behaviors. ¿From the previous considerations it appears evident that this approach could be not completely satisfactory when dealing with the organism's complexity. Indeed, already from a pure physical point of view, it has been stressed that the kind of explanations that we must employ when dealing with complexity is not focused on determination of single trajectories or behaviors but rather on classes of behaviors sharing some common general property [32].

So, I would like to introduce the notion of teleonomic mechanisms when the inputs of the system under consideration are insufficient to determine its future state without additional inputs coming from the environment and it can follow different and alternative paths in order to reach the same final state. So, while in mechanic causation only the antecedent state matters, in teleonomic causation only the final state does (for instance the species-specific state to be reached at the end of development). In other words, mechanic causality is sensible to past conditions but is robust in the ability to produce a certain effect given certain conditions (this is the determinism of classical systems). Teleonomic systems, instead, are robust relative to the final state or the task to be accomplished: Many variation of the past conditions will not affect the realization of the task or the arrival to the final state.

\subsection{Explanatory Power}

Let us first understand which are the conditions for having teleonomic causation. I have already mentioned complexity, an issue that will be discussed also below. Another condition is a sharp separation between self and non-self. As mentioned, this is what allows the organism to exercise a control upon the entropic fluxes between itself and the environment and is an important difference relative to any inorganic self-organizing system, which cannot control these fluxes and is therefore directly dependent on environmental parameters. With self I mean the systemic totality of the living organism, except all what lies outside of it or does not fully depend on this systemic organization. Organisms need not only to accommodate to the external environment (to a certain extent also abiotic physical systems do the same by reaching a certain equilibrium state relative to environmental parameters), but, since there is now a boundary between self and non-self, there is a systemic self-referring circle of reactions and control mechanisms, such that finding the own place in the environment splits in two different directions: Accommodation of the organism to the external environment and assimilation of the environment by the organism. When I say assimilation, I am not only speaking of feeding but of the ability of any organism to carve out the environment according to its needs. As we shall see, very often we have a combination, an optimal trade-off, between these two opposite processes. In this way, any organism maintains its 
independence from the environment not through absence of change but precisely through change, as beautifully understood by J. Woodger [33]. The separation between biological self and non-self has been made possible by the building of a lipidic membrane in primitive organisms able to segregate an autocatalytic molecule with informational value.

Let us now understand the kind of mechanism at work in teleonomy. Teleonomy can arise only when a circuit is established in which three types of feedback can work together in an appropriate way:

(1) A self-increasing feedback mechanism, coming from the inside of the organism, directed to the building or growth of structures in phenotype or of the three-dimensional configuration of a protein,

(2) A negative feedback [34], whose ultimate source is in some environmental perturbation, mainly addressed at blocking the expression of the activated DNA or at having other damping effects on the phenotype, and

(3) An anti-feedback directed toward the environment, that is, a process spontaneously activated by the organism to prevent dangerous environmental effects and to restore its own homeostasis. This was well understood by Bichat [35], who spoke of an internal principle of organisms able to counteract the spontaneous tendency to destruction.

It is evident that several environmental cues can also induce DNA expression, and in this way they appear to give rise to positive and not negative feedback. However, this is the effect resulting from the ability of the organism (thanks to the feedback-control mechanisms) to turn a potential threat for its homeostasis into a source of stability and growth. Not only significant aspects of development but also the whole mechanism of self-reproduction, especially in the cycle of higher eukaryotes, is a teleonomic mechanism.

Summing up, teleonomy is a mechanism based on the attraction exercised by a "final" or next stable state on a biological system. Actually, at least in the most elementary situations, the final state of the system is not a reality existing by itself outside the system and before the system implement it. Nevertheless, the crucial point is that it is able to regulate the dynamics of the system from the inside, and in this sense it is something more than a purely formal constraint acting only from the outside, as it is still true for any other physical system. The system is able to do so because its dynamics has been selected (through natural selection) in such a way that the organism already possesses, embedded in the cluster of relations characterizing it, the potential resources to deal with whole classes of external and future events in order to eventually reach the next stable state (the attractor of the system). Therefore, the system can establish a channel with the environment even if in fully dynamical independence from it. This is the reason why, when certain external signals occur (within a certain tolerance window), the organism is able to properly react and even to integrate them. This plays a relevant role during developmental processes, since any organism is prepared for specific environmental stresses in an anticipated way [36]. Therefore, this relation with the external cues and events makes these cues or events able to concur in determining the final output of the dynamics as if it would be an outside force acting on this dynamics. Here, something that comes later on concurs to determine a process from the start that explains and justify the presence of that character itself. Even if attractors also play a role in pure classical dynamics, 
they are especially relevant when complex system are involved, in which various feedback mechanisms play a major role.

It is teleonomy that accounts for the distinctive way organisms deal with environment during the generations and especially for the co-adaptation between organism and environment. Indeed, since the selected co-adaptation between organism and environment is so complex and somehow fragile, any genetic mutation and especially its expression in the phenotype (or in protein building) cannot be too abrupt. In other words, it must not perturb this precarious balance. It must necessarily result in a further growth or different usage (or even use in a different context) of already existing structures. This explains phenomena like exaptation [37], but also the general rule in biology that there is never a new starting but always a further development of already existing structures. Often, it has been asked why nature does not work like an engineer, who is able to project a machine in a ordered way instead of using old existing structures. ¿From an ideal point of view, this is preferable, since it allows to have a machine that works better at a lower cost. However, this is possible because it is supposed that the engineer has full control on the conditions for generating that machine (ability to plan, availability of materials, of the mechanical parts or ability to produce them if they fail, money for doing all that, market interested in that production). However, organisms are not totally autarchic and depend on the integration of environmental cues, and the environment is in principle unpredictable.

\section{A Selection System}

\subsection{The Membrane}

An interesting system combining complexity and information is the selection system of an organism, which in unicellular organisms is constituted by the membrane. The DNA does not code for the membrane, but only for the proteins building or regulating the membrane (and obviously for the gate proteins in the membrane). The membrane, playing the function of a decider (either allowing or preventing something to go in or out of the cell), has been evolutionary built from lipidic material through pure physical mechanisms and, in the transmission to further generations, it is not subjected to genetic variability (it is a piece of physics recruited by life): Omnis membrana e membrana [38]. In other words, the lipids constituting the membrane are the only chemicals that do this work and therefore have essentially the same structure as they did in our ancestors, i.e., they cannot be modified by the genome. The independence of the membrane from the genetic system is very important and even necessary, since any true selecting system must always incorporate a random element. In the case of the membrane this is not only because this structure does not depend on the genetic system, but also because it is independent from the external environment, being that it is rather a shield against it.

To be more specific, the membrane is a lipid bilayer (constituted by phospholipids, glycolipids, and cholesterol) showing hydrophobic tails sticking together while the hydrophilic heads remain in contact with water. Membranes spontaneoulsy generate sacklike vesicles, that is, relatively closed systems. This is a self-assembly process.

The membrane proteins, allowing for both transduction and entropic fluxes from the interior to the exterior, are an example of allosteric proteins, that is, of proteins that can be in two different states (for instance, allowing or not allowing some ions to enter a cell), according to whether or not they 
are phosphorylized or dephosphorylized (phosophatase) [39-41]. Very important insights into these mechanisms were brought to light by considering the family of the phosphoinositides, which are very relevant, among other things, in cell communication [42-44]. The lipid tail of these molecules renders the phosphoinositides obligately membrane-bound. This makes them well-suited for marking particular membrane partitions. Phosphoinositides achieve direct signalling effects through the binding of their head groups to cytosolic proteins or cytosolic domains of membrane proteins. Thus, they can regulate the function of integral membrane proteins, or recruit the membrane signalling components.

\subsection{Information}

The membrane (understood here as the whole complex that regulates the fluxes between the exterior and the interior) is a true selection system in an organism and in this way allows for information control on the external environment. Evidence for this is the fact that the most important protein responsible for vision (rhodopsin) can also be found in bacteria embedded in the lipid membrane or in some mobile algae, with the exact same photodetection function. Therefore, the root of information codification and processing of any more sophisticated sensory system is the information selection of the membrane.

To understand how this system works, we need more general considerations. The organism as a system and an individual unity represents the complexity that deals with physical perturbations in the external environment and is able to transform this in specific appropriate actions (or reactions) that are congruent with the goal of self-survival. The membrane selective system is somehow the bridge or the interface between this external perturbation and everything may happen thereafter in the organism. It is what transforms this external perturbation (through transduction or, in higher eukaryotes, through particular sensory channels and organs), which in itself is a pure physical phenomenon, into codified information generating appropriate inputs that are then sent to the metabolic system where they are combined with further endogenous inputs. Obviously, in unicellular organisms the membrane also has another significance, that of being the device allowing for the coming in or out of elements that are necessary for the metabolism. This is related however to the actions that the organisms are able to perform after the codification has already happened and the information has gone through the whole organism. We should not mix these two very different issues, which in higher organisms become fully separated.

The cellular transduction consists of:

(1) A signal triggering the whole process (the so called first messenger) that in most cases binds a receptor (gate protein) without entering itself into the cell;

(2) As a consequence, a second messenger (an intracellular molecule) is delivered inside the cell and may produce amplification of the initial signal (a sort of cascade); this can be assumed to be the proper codification step;

(3) Finally, there is an activation of a response going into the inside of the cell to the metabolic system.

Without considering now what further steps there are inside the organism, let us understand what the significance is of this codification-selecting system. Each codified input somehow represents a certain surprise. The surprise expresses the novelty represented by the codified input originating from the 
external physical perturbation. In other words, it is the mismatch or distance between the current state of the organism and this input information. Now, the point is that this mismatch is coded in the selection system itself in the form of a mismatch between the state of the receptor (which stands here for the whole organism) and the new input information. Thereafter, the selection system informs the metabolic system about this kind of situation. The metabolic system in turn, takes this information, together with other endogenous signals, as a sign to give rise to another series of procedures to preserve or restore its metabolically homeostatic state. In other words, an occurrence of information selection (coding and response) gives rise to a whole procedure, having a final thermodynamic and entropic character.

Mathematically speaking, we can express this procedure by employing a variable $i$ which represents the new input as a function of some external (environmental) parameter $k$ and as describing (determining) the state of the receptor of the sensory system [45-48]. It is interesting to remark that this powerful formalism has originally arisen in the context of neurosciences. What I am trying to show here is that it can be generalized to apply to any organism. The quantity $A$ represents the action that the organism undertakes in order to minimize the surprise given by the conditional probability

$$
p(i, k \mid A)
$$

It is important to understand that the action can be taken both on the environment or the organism itself. To be really efficacious, however, such an action always implies an action on the external environment as a way of avoiding further surprising inputs. Now, it can be shown that lowering the surprise (or surprisal) means to lower the following quantity that is an informational analogue of the free energy in thermodynamics [49]

$$
g=-\langle\ln p(i, k \mid A)\rangle_{p^{\prime}}+\left\langle\ln p^{\prime}(k ; s)\right\rangle_{p^{\prime}}
$$

which implies that this lowering is in full accord with general statistical laws and can even be considered quite natural. The two expressions mean some (statistical) mean value of the relative logarithms of probabilities. The quantity on the right represents the mean value of a log-probability distribution of both the environmental parameter $k$ and of the internal parameter (state) $s$ of the organism. Obviously, there can be many factors contributing to a certain input information. However, this complication is unnecessary in such a context. Moreover, this distribution is always positive. The whole expression can also be reformulated as

$$
g=-\langle\ln p(i \mid A)\rangle+D_{K L}\left(p^{\prime}(k ; s) \| p(k \mid i, A)\right)
$$

where the second term is the so-called Kullback-Leibler divergence (also called relative entropy) that here measures the distance of the probability distribution before the two vertical lines from the conditional probability of the external parameter $k$ given that there is a certain input and a consequent action (after the two vertical bars). Given two probability distributions $p(j)$ and $p^{\prime}(k)$, the classical Kullback-Leibler divergence (in the discrete case) is given by

$$
D_{K L}\left(p^{\prime}(k) \| p(k)\right)=\sum_{k} p^{\prime}(k) \lg \frac{p^{\prime}(k)}{p(k)}
$$

Note that the mutual information

$$
I(J: K)=H(J)+H(K)-H(J, K)
$$


can be expressed as the Kullback-Leibler divergence between the joint probability and the product distribution of two involved parameters. Strictly speaking, surprise is the first term in Equation (14). The surprise is implicitly conditioned upon the organism in question. It can be seen that by minimizing surprise one is effectively maximizing the probability of the selected inputs under a particular action (or state of the organism). In other words, lowering the surprise means to choose a "model of the world" with the smallest $g$, whilst the latter has the highest marginal likelihood. This follows because $g$ is an upper bound on surprise, given that the Kullback-Leibler divergence is non-negative.

Therefore, minimizing the expression (13) amounts to minimizing the negative log-probability of the sensory input (reducing the mismatch between the expectation and the input) [50]. This is precisely what we expect any organisms to do: It will expose itself selectively to those causes in the environment that it expects (or is programmed) to encounter. However, these expectations are limited to the repertoire of physical states that the system can occupy by preserving its homeostasis, and therefore the net result is that the inferred causes approximate the real causes.

The crucial point to understand here is that, in statistics, the minimization of the surprise is equivalent to a Bayesian probability computation, where the function $g$ above can be used to approximate the likelihood function, i.e., the probability that, given a certain transduction, the parameters that may have caused it are those that the organism expect:

$$
p(k \mid i)=p(k) \frac{p(i \mid k)}{p(i)}
$$

where $p(k \mid i)$ is the likelihood function that the signal $k$ has indeed given rise to the input $i$ (for the sake of simplicity I do not consider the action $A$ here), $p(i)$ is the a priori probability of the input (here, I do not consider internal parameters for the sake of simplicity), $p(i \mid k)$ is the a posteriori probability of having the input $i$ given the parameter $k$, and $p(k)$ is the probability distribution of the environmental parameter $k$.

\section{Teleology}

\subsection{Conditions of Teleology}

By teleological causality I mean the mechanism through which a system exercises an informational control on another system in order to establish an equivalence class and select some specific information for its metabolic needs. In other words, it is a semiotic process through which a system refers to another external one in relation to its own goals, which are informationally determined and thermodynamically supported. It was the historical merit of F. Ayala to have stressed that teleological explanations are appropriate and indispensable in biology, and cannot be reduced to non-teleological explanations without loss of explanatory content; although at the same time they must not be in contradiction with mechanistic explanations [51]. Moreover, Ayala was the first scholar to use both the concepts of teleonomy and teleology as explanatory tools for biology [52].

Teleology should have arisen through some teleonomic mechanisms thanks to natural selection. The most elementary goal of a biological system is its own self-maintenance. A complex system that needs to rule in a certain way the entropic fluxes with the environment is naturally pushed (through natural selection mechanisms) for the sake of its own self-maintenance to act on the environment according to 
certain internal needs (i.e., which depend far more on its own self-organization than on environmental parameters) [53].

Figure 7. The constitution of a three-amino acid sequence. The part in blue is the amino group, the black carbon atom is the central atom with an added hydrogen atom (also in black), the carboxyl acid group in red, and the side chain in green. Note the repetition of the structure on the left and on the right of the peptide bond until the carboxyl group end.

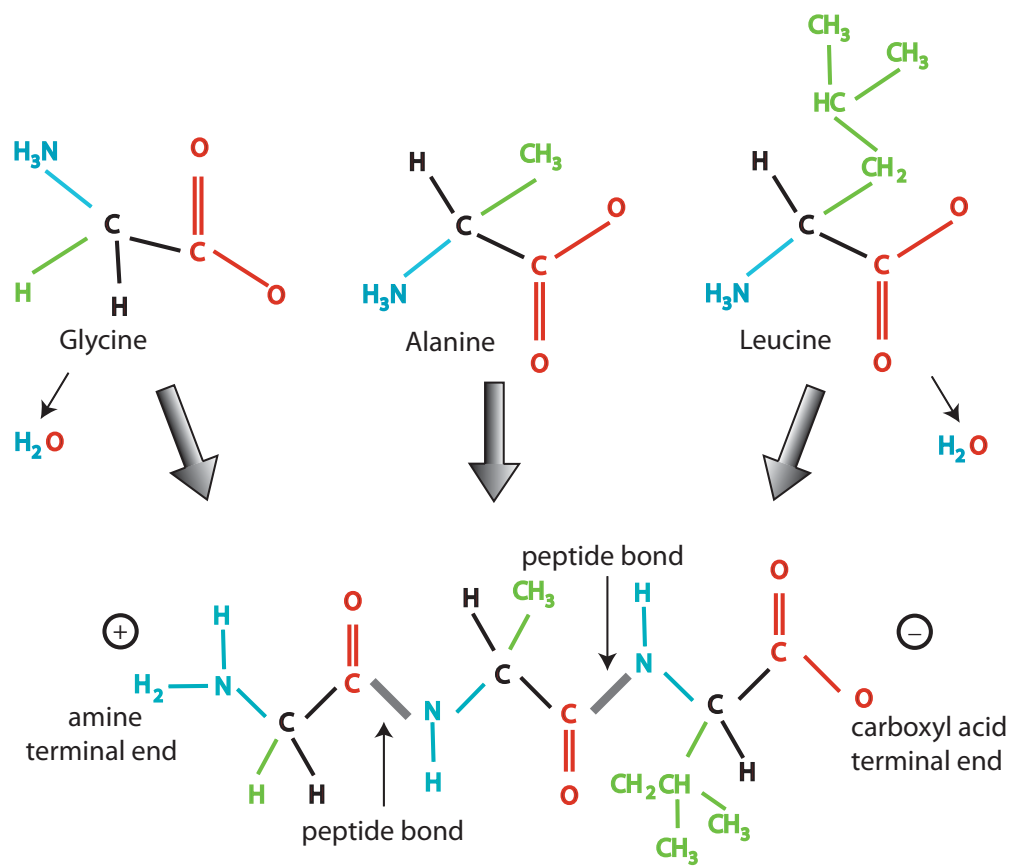

There are several conditions of teleology. The first one is a certain separation but also connection between information and function. I have said that DNA codifies information and RNA represents a set of instructions finally giving rise to the protein. A protein no longer represents information in any sense. In DNA, apart the backbone, we only have hydrogen bonds between informational molecules (bases). In tRNA, we have a chain of informational molecules plus some hydrogen bonds between them. It is important to understand that already RNA can show hydrogen bonds that do not fulfill the requirements of information codification. RNA is indeed a macromolecule that can also have a function like a protein. The primary structure of a protein is constituted by single peptides connected by peptide bonds, i.e., a carboxyl group of a molecule reacts with the amine group of another molecule releasing a water molecule. It is evident here that the only element that distinguishes the different segments is the side chain [Figure 7]. Now, the relevant point is that these segments can no longer be detached and recombined as we expect from true codified information. I recall that it is the latter property that allows DNA and RNA to be good chemicals for dealing with information [Figure 1]. In the final stage, the protein folds thanks to non-covalent bonds (hydrogen bonds and van der Waals forces). Here, one could distinguish between concatenative compositionality, typical of the classical information approach, and a merely functional compositionality [54]. Concatenative functionality preserves tokens of an expression's constituents (and the sequential relations among them) in the expression itself due to the linearity of information codification. The reason why they are so common is that they are also functionally compositional. But most functionally compositional schemes are only functional 
and not concatenative. This is a specific modality of protein building, especially in its tertiary and quaternary structures.

The whole process leading from the codified information in DNA to the protein is irreversible. If it would be, we should have at least one case in which it is also invertible (at least for statistical reasons). However, no protein can back-translate itself into a RNA that could reverse-transcribe into DNA (even if reverse transcriptase is well-known). Proteins can allow expression of a gene or block its expression, can have a role in translating (other) proteins, but cannot back-translate themselves recovering the information that started the process of their own building: This would be an instance of information control on their own building process and the reason for its non-occurrence is the impossibility to back-transform a functional unit (the protein) into the codified information that gave rise to this unit, since this information has been lost precisely in the process through which the functionality has arisen. It is known that the sequence of amino acids in a protein can be reconstruct with a method known as automated Edman degradation [55]. It is important to understand that this is not an easy work and that in many cases we can succeed in such an extrapolation only by using a recombinant DNA technology, in which long stretches of DNA are cloned and sequenced to reveal the amino acid sequence of the protein encoded by the gene (it is a complementary experimental approach). In other words, we can recover the amino acid sequence by using additional codified information. All that also means that information transfers from protein to protein, from protein to RNA, and from protein to DNA are all impossible processes. The conclusion is that the central dogma cannot be violated [56].

Figure 8. From information to the function: The two roots of biology: Linearity and complexity.

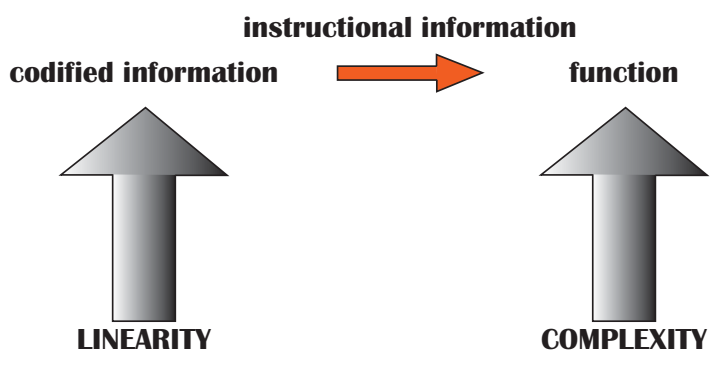

It is therefore important to understand that there are two different roots of biology: The linearity of information codification and complexity. Although complexity is also important for DNA and RNA, it is constitutive for proteins and their functions [Figure 8]. Let us consider a little bit the concept of biological function. Any biological function has the following general characters:

- It cannot be reduced to pure structure, even if it depends on some specific structural elements that are crucial for the function and on the architecture of the whole. Hubs in networks are a manifestation of this property [57,58], something that very much contributes to the robustness of both networks and functions.

- It is always instantiated in some operation: However, it does not depend on it but rather on some formal constraints from above (ultimately, from the needs of the whole organism) and the goal of this upper level. With operation I understand any spatially and temporally coordinated pattern of physical-chemical interactions (it is a complex structure) able to fulfill a function. Operations 
are therefore a bridge between chemistry and functionality and display both efficacious work and variety.

- It is connected with the needs of the whole organism and therefore relies on the cooperation of different subsystems, like the genetic, metabolic, and selecting systems.

- It depends on information codification but is not its immediate result. The work of natural selection is to have selected the whole process leading from DNA to protein and not single segments.

A function cannot be accessed or grasped from the pure local level of chemical interactions: We need to compare different operations in the same organism or in different organisms in order to ascertain that they are functionally equivalent and in this way to grasp meaning and character of the related function. The latter does not violate chemical laws but it represents and helps notwithstanding a further level of organization than the level of operations and chemical interactions.

This means that functions can be understood as equivalence classes of operations, showing a property that we have already found in the genetic code: degeneracy [59]. This is a characteristic difference relative to mechanical causation. To understand this, let us take the example of a thermostat. Although thermostats have feedback circuits, these circuits are designed and built by humans and have no goal in themselves. In some specialized literature one often speaks of a the goal of a certain artificial device. However, this is only a figure of speech, since the goal is only in the humans that have built it. The "information control" exerted by a thermostat (maintaining a certain temperature) coincides here with a single path of physical interactions (the switch-mechanism is turned on and off according to the temperature level attained). In other words, a mechanical device like that can only work in the way it has been arranged by humans. Instead, in a system with true goals, there are in general several—physically or chemically different - operations that can lead to the same desired outcome (within a certain tolerance window). Now, any organism shows an incredible ability to keep chemical interactions under control to deploy the operation (that, in certain conditions, is appropriate to realize a functionality) but also to switch to another operation if the former is impaired or somehow blocked [60]. This is shown by some experiments proving that the functionality can be preserved by interchanging crucial biochemical elements [61,62].

Equivalence class is a rather formal concept that can be commonly found in many fields, whose properties are

- Symmetry: If the item $a$ is equivalent to the item $b$, also $b$ is equivalent to $a$,

- Reflexivity: $a$ is equivalent to $a$, and

- Transitivity: If $a$ is equivalent to $b$ and $b$ to $c$, also $a$ is equivalent to $c$.

However, when speaking of equivalence at the most basic level of molecular interactions as in the DNA-RNA-protein circuit or at the level of the molecular mechanisms ruling the selection performed by the membrane, we have to do with functional equivalence classes. Functional equivalence class is context sensitive. It is a pure biological category, where different operations are considered equivalent if they produce the same outcome for some functional need (the goal). Thus, I focus here on 
functional equivalence classes rather than purely formal equivalence classes, even if the formal properties previously defined must also hold for them. This is however not in an absolute sense: Since operations equivalent with respect to a certain function are not automatically equivalent for other functions, it is important to identify unequivocally the function (and therefore the context) concerned.

Figure 9. E. coli's movement. Above: Straight swim. In this case, the flagella turn counter-clockwise. Below: Tumbling. In this case, the flagella turn clockwise.

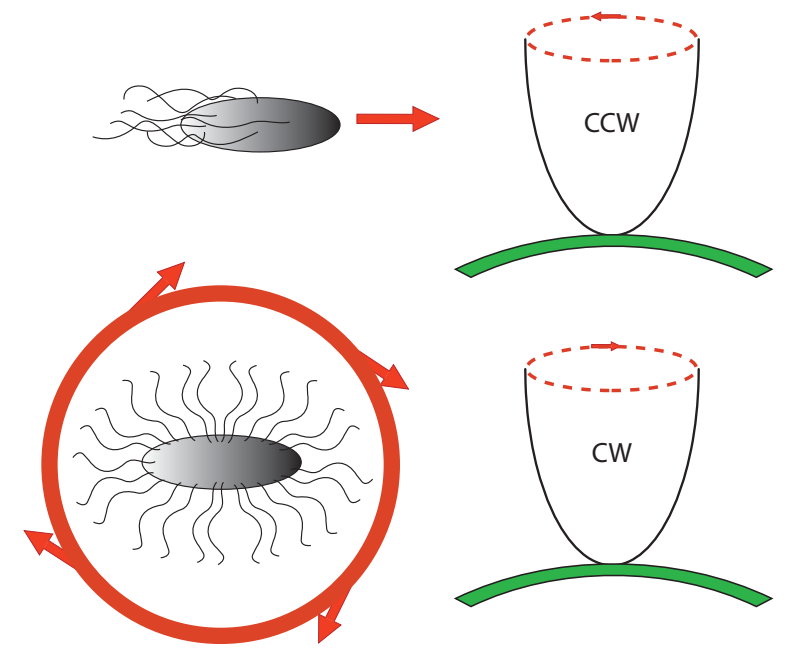

\subsection{Chemotaxis as Example of Teleology}

Now, we have information control when an organism is able to individuate, select, and use specific signals (by treating many signals as equivalent) for displaying certain vital functionalities. This is particularly evident when the organism picks out certain environmental signal for monitoring certain environmental parameters and execute certain functions. A very relevant example is shown by the chemotaxis of the the bacterium Escherichia coli $[63,64]$. Such an organism is unable to choose by itself the direction of swimming. Moreover, it is unable to preserve a straight movement for more than few seconds due to the fluctuations of the external fluid. These bacteria alternate tumble and swim phases. In presence of a chemical gradient, they will base their motion on this parameter. If this organism senses to swim in the right direction, it will preserve straight line as long as possible before tumbling. If, on the contrary, it senses that it is swimming into the wrong direction, it will tumble sooner. It is a sort of induced "choice". The helical nature of the single flagellar filaments allows these two types of movement [Figure 9]. Let us now consider a little bit the response mechanism.

Chemical gradients are sensed through multiple transmembrane receptors, called methyl accepting chemotaxis proteins (MCPs), which vary in the type of molecules that they detect [65]. These receptors may bind attractants or repellents directly or indirectly through interaction with proteins of the periplasmatic space between the exterior and the interior membranes. The signals from these receptors are transmitted across the plasma membrane into the cytosol, where Che proteins are activated. The Che proteins are able to alter the tumbling frequency, and alter the receptors [Figure 10]. Signals are codified and passed from the transmitter module of one protein to the receiver module of a second protein via phosphotransfer. In the involved pathway, a family of related transmembrane receptors 
act as the input module by binding either small chemotactic molecules or their periplasmic binding proteins. Once these effectors are bound, the activity of a transmitter histidine kinase (CheA) that is associated with the cytosolic domain of the receptor(s) is rapidly modulated. Changes in the activity of this kinase lead to transient increases or decreases in intracellular levels of phosphorylated CheY (the response regulator) which directly affects flagellar rotation and the frequency of their reversal. Slower habituation of this response, effected at the level of receptor signaling, is induced by the reversible methylation and demethylation of a specific group of glutamate residues within predicted coiled-coil regions of the receptor cytosolic domains. These covalent modifications are catalyzed by an S-adenosylmethionine-dependent methyl transferase (CheR) and a partner methylesterase (CheB) that act to respectively increase or damp the signal. $\mathrm{CheB}$, which is another response regulator, is also a substrate for CheA kinase activity. The protein is most active as a methyl-esterase in the phosphorylated form and further serves as a feedback regulator of signaling.

Figure 10. Activated forms of the proteins are shown in darker color and solid arrows are used for indicating activation. (a) The high level of phosphorylated CheY increases the frequency of switching to clockwise flagellar rotation and thus determines tumbling. (b) When a receptor binds ligand and/or is unmethylated CheA is inactive. The levels of phosphorylated $\mathrm{CheY}$ are reduced leading to more counterclockwise flagellar rotation and more running. With CheB inactive, the methyltransferase activity of CheR (purple) serves to decrease receptor sensitivity. Adapted from [65].
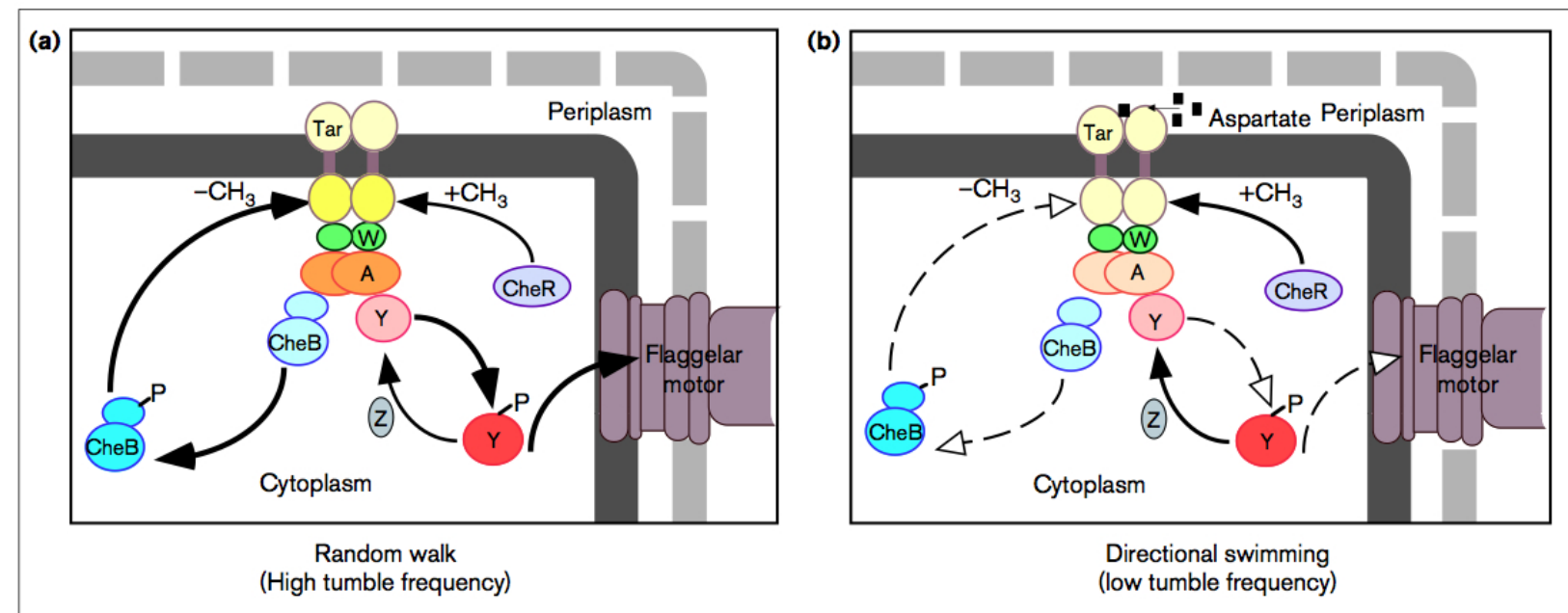
Figure 11. The sensory component processes an environmental signal through its input module to activate the transmitter module. Phosphoryl transfer from the transmitter to the receiver-comparator module of the response regulator component, jointly with other inputs coming from processes inside the organism, activates the output module, and therefore triggers a final motor output.

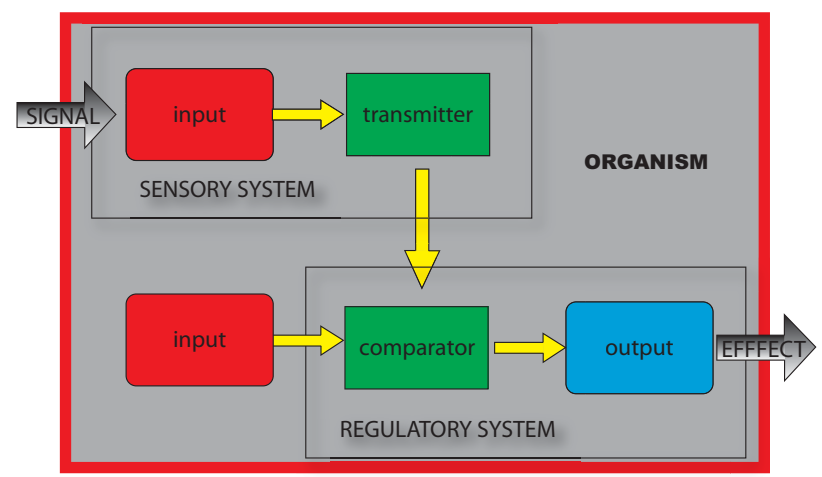

Apparently, this behavior is quite mechanical and does not seem to display the elements that we expect from a true information control. However, this is not the case. The first point to stress is that here also a regulatory component is involved [Figure 11], determined by the opposite effects of the proteins CheB and CheR. This prevents us to consider the whole as a pure input-output mechanical engine. Anyway, this does not suffice for establishing information control. Here the two crucial questions are: Is a true comparator nested in this regulatory part? Is there some form of information codification here? When we have a more precise look, we shall remark that

- To make temporal comparison of chemoeffector levels (a pure informational operation), the bacterial cell requires a sensory adaptation mechanism that cancels chemoreceptors signal outputs in static environment, no matter what chemoeffectors may be present [66] (whether attractive or repulsive). This enables the bacterium to reset the threshold sensitivity of the signaling system in order to detect any new change in the chemical environment. It is a true information-erasing mechanism necessary for information acquiring, a kind of very elementary representational function.

- Another very important element is reaction timing: Because Brownian motion of the fluid medium can randomly reorient the bacterium, this requires very short response latencies. It is here that genetic (instructional) factors play a role by enhancing and damping protein production. It has been indeed observed that the protein CheZ plays a very important role in enhancing the rate of CheY (which is directly involved in the change of motion) dephosphorylation [67]. Moreover, in the whole regulation also the transcription factor NarL plays a role.

This means that the regulation and selection activity is indeed very sophisticated and that informational aspects are involved in signals transduction and information erasure, comparison of chemoeffector levels, DNA activation or repression. This also means that several comparisons are executed along the whole path going from transduction to reaction: Between concentration levels of nitrate or phosphates and the required ones, between the proteins at disposal and the required ones, and so on. The single segments and operations can be obviously explained in pure chemical terms. But the whole pathway or network 
here involved, in which also informational aspects play a major role, cannot and this is an instance of information control on the environment.

\subsection{Epigenetic Processes}

The evolutionary significance of development is related to the fact that young organisms exhibiting structural and behavioral development survive and reproduce better than other organisms whose behavior changes less considerably with age and with size. For this reason, epigeny is essentially a compromise between the continuity of the species and the discontinuity of novelties. To understand this can bring us to a deeper understanding of epigeny:

- For the reasons indicated previously, epigeny can indeed be understood as a compromise between cell proliferation (the continuity of the self-reproduction and the own self-production) and the necessity to control cell division (discontinuity, consisting in negative feedback against growth), especially of some specific cells. This has obviously an evolutionary significance [68]. Let me add in this context that early metazoans blocked proliferation by differentiating in a ball of ciliated cells, a primitive blastula [69]. The only cells that could still differentiate were those already in or migrating into the inner cavity of the ball, a sort of gastrulation. In this way, cilia also acquire a developmental role: They help in maintaining the animal shape.

Figure 12. A very schematic drawing of the main typologies of epigenetic interactions: The gene $\mathrm{A}$ is able to activate genes $\mathrm{B}$ and $\mathrm{C}$, which in turn respectively activate genes $\mathrm{D}$ and $\mathrm{E}$, on the one hand, and $\mathrm{F}$ and $\mathrm{G}$, on the other. Any gene downwards negatively feedbacks on upstream genes. Genes located at the same level of the activation cascade influence reciprocally with waiting signals for regulation and fine-tuning of the process.Interestingly, genes $\mathrm{E}$ and $\mathrm{F}$ may be activated synchronically (and in this way share information) even their parent genes B and C are not causally sequential.

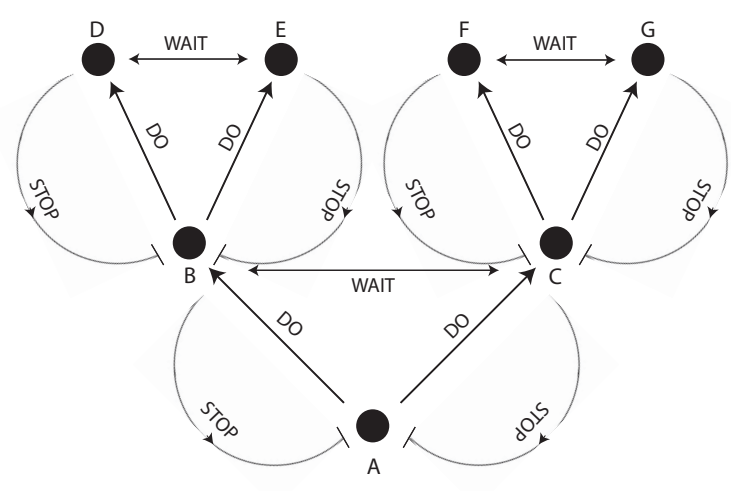

- Epigeny can be understood also as a compromise between environmental inputs (the discontinuous aspect here) and control genes providing anti-feedback (the continuous aspect, from the point of view of the organism). Environmental factors like temperature, nutrition, pressure and gravity, light, presence of predators, presence or absence of conspecifics are very important for the development of an organism. The action of the environment on the organism can be on 
transcriptional regulation, on the neuroendocrine system, or be a direct cellular induction: They correspond to an action on the genetic, metabolic, and selection system, respectively. The presence of these factors explains why epigeny is not a full teleologic causal process but also consists of considerable teleonomic processes. However, this does not mean that the environment somehow guides or instructs the organism. The environment only provides negative feedback. It is thanks to those teleonomic processes that the organism is able to canalize and even make positive those environmental stimuli for its own growth.

- Continuity and discontinuity are also important from a further perspective. It is perhaps convenient to distinguish between initial information (the continuity across the generation) and information conditioned by the specific metabolism of an individual (the discontinuity). An evidence for this is the fact that differences in position and surroundings in presence of a faithful genetic duplication that result in differences in phenotypic patterns are regulatory differences that are responsible for divergences in developmental processes.

It is crucial to understand that epigeny is based on:

1. Cellular memory: Initially, memory almost completely consists of genetic information; as epigeny goes on, epigenetic memory progressively grows. It is, therefore, a parallel process and, to a certain extent, also a cyclic (wave-like) one.

2. Cellular machinery, which in turn also depends on cellular memory. Every cell starts its own version of life anew, since its configuration depends on (both temporally and spatially) local context much more than on the genetic information it brings (this represents the point-like, discontinuous aspect).

The ability of a cell to detect and react to a specific location thanks both to its memory and its current state is called topobiological potency (another form of potential information). For this reason, development cannot be understood as a mere sum of cellular behaviors but it is a very complex feedback network in which regulatory processes are at play. Also from another point of view, epigeny presents both continuous and discontinuous aspects: Each generation event and each environmental input are discontinuous from the point of view of the epigenetic control. Instead, the developmental program is the continuous aspect.

Phylogenetic transmission is a divergent process in its own essence (new species always arise), even if convergent aspects play also a role. Ontogeny, understood as the whole life trajectory, is basically a convergent process. Epigeny, in its own nature, is a convergent-divergent increase of complexity [70] [Figure 12].

During epigeny there are many possible paths that can lead (converge) to the same species-specific result, a behavior called epigenetic degeneracy. Degenerate systems are different from redundant ones, since the latter are systems with complex and perhaps incompatible homologous representatives, while the former ones produce clearly identifiable homologues. Epigenetic degeneracy means that the initial conditions given by the genetic memory of the system, its initial state, and a given environment do not suffice to single out the developmental path of an organism. However, I recall that, when the organism approaches more and more to its mature form, it will become more and more difficult to change the 
followed path, and the danger that any change may turn out to be disruptive in development becomes increasingly strong.

Obviously, also the opposite is true, that is, a single gene, through epigenetic mechanisms, can give rise to a set of different ontogenies when it is exposed to internal or external environment. I recall that this effect has been called developmental reaction [71]. Wright [72,73] was the first to understand that there is not only a set of effects of single genes, but that these circuits are actually constituted by a network of interacting genes (epistasis). Therefore, gene complexes have coadapted in the course of evolution. In this way, evolution does not present a single peak in the fitness landscape but multiple peaks of various heights.

Summing up, interactive (organism-environment, cell-cell, chemical aspects-genetic regulation) dynamical processes are crucial here and the final (mature) species-specific steady state attained at the end of development is an attractor: We have a dynamical basin of attraction in which the whole information necessary for joining the attractor is not present from the start as a set of sufficient instructions [26], but is rather a process of self-organization through which a complex system comes out starting from some initial instructions and the attractor itself may be changed during this dynamic process:

(1) The set of initial instructions [Section 2] allows for the building of the first elements that give rise to a cascade process (positive feedback) through which further genes and signals are activated and propagated.

(2) The cellular multiplication process proceeds by a successive and parallel but hierarchical building of different levels of commands and containment (body plan, organs, tissues, single cells), where negative feedback and anti-feedback effects are at work. The process is governed by a principle of information accessibility allowing for different levels of information encapsulation, as shown in Section 3.

(3) During this process, many events happen that have multiple effects establishing new interconnections and therefore a huge network of shared information, both horizontally and vertically [Section 4].

(4) The organism is characterized by the fact that it actively searches for the environmental cues (temperature, light, foods, and so on) that allow its own development [Section 5], and it is here that (through anti-feedback) information control and teleologic causation come into play (as explained in this section) assisting epigeny in the process (cognition assists epigenetic processes).

(5) The whole can be seen as a process tending to a final stable state through a trajectory where the distance from the final species-specific steady state is minimized through the active concourse of the organism. However, since each stable state is provisional, we have an itinerant dynamics.

The problem of the increasing complexity in epigeny can then be reduced to a very specific problem of modularization and integration: It is this difference in information (between the memory and the current state as well as between the current state and the final state), in an opportune teleonomic network transforming the mechanical inputs in a controlled set of instructions, that needs to be further implemented in an interactive cascade process. 
In the introduction I have listed some of the fundamental concepts that should contribute to a further progress in biology. These concepts are fully involved in the way epigeny occurs. I have spoken of formal constraints: The whole process described above goes on thanks to those constraints (for instance, those stemming from the hierarchy of epigenetic processes). The process and the different states reached at different times show degeneracy. The whole is characterized by information sharing among different subsystems and cells, and information selecting inside a subsystem or a cell. The differential timing is here crucial in affecting the way in which both single subsystems respond to certain stimuli and the whole body plan emerges. Events producing local energy dissipation reinforce or weaken interconnections, thus enabling the proceeding along a certain epigenetic trajectory or the plastic switching to alternative paths, if required.

\subsection{A Generalization}

Therefore, any organism (through both teleonomic and teleologic processes) tries to dynamically integrate as much as possible any environmental perturbation in the web of its regularities. Due to the generality of both the selection and the correlation principles, my guess is that this responds to a general behavior of nature, that could be summarized as

In appropriate conditions, any system is pushed to minimize the distance between the selected option and the less expensive one or to lower the level of perturbation of its constitutive web of interdependencies provoked by the selection.

This principle means, in particular, that the organism maintains its independence from the environment precisely through changing. Indeed, the perturbation, being out of the system's control (otherwise, it would not happen at all), cannot ever be completely washed out. This means that the system will never fully recover the previous equilibrium state. Therefore, it is a general principle of dynamicity. To this extent, life is deeply rooted in thermodynamics (even if the bridge between entropy growing and entropy lowering processes is constituted through an informational step). Indeed, the Le Chatelier-Braun principle [? ] states that any perturbation of a factor contributing to equilibrium induces a compensating change in an opposing factor. The specificity of organisms is, however, in the fact that such a dynamic integration is or can be adaptive, that is, a change for the better. The concept of itinerancy, which is very widely used in the theory of complex and chaotic systems can be very useful here helping us to overcome the traditional fixed-point attractors in optimal control theory.

\section{Conclusions}

For a paradigmatic shift to occur in science, a new conceptual framework must allow to individuate different explanatory mechanisms more appropriate than the previously established ones with respect to the object of study and the related open problems.

To do that, we need to consider the problem in its complexity. The three dimensions treated in this paper are: information, complexity, and function. In the introduction I have said that the theory of complex systems did not succeed in changing the approach in biology, as sought by some scholars. The reason is that complexity alone cannot provide for such a conceptual change since it is unable to display new explanatory mechanisms. As a matter of fact, very often we employ simulations when dealing with 
complexity. Instead, when we consider the above three dimensions together, the possibility arises to find such mechanisms. I have indicated them as teleonomy and teleology. They are non-mechanistic mechanisms, if I am allowed to say that. They have arisen thanks to the cooperation between natural selection and formal constraints, due to informational requirements, complex correlations, and the necessity to implement appropriate functions to survive. The old understanding of natural selection as acting only on single aspects or features is a heritage of classical mechanics. If we only consider the immediate action of natural selection, this is even right. However, if we consider the total effect of natural selection, this contradicts the facts we know. Indeed, thanks to those constraints, the organism shows a teleonomic process of co-adaptation with the environment allowing the emergence of new and appropriate functionalities. Moreover, the organism is also able to control and modify the environment thanks to teleologic processes, which backreact on its own evolutionary path.

A further point to be stressed is that what I have called accommodation of the organism to the environment has its root in teleonomy; whereas, assimilation of the environment by the organism has its root in teleological processes. Teleonomy and accommodation, on the one hand, and teleology and assimilation on the other, should be regarded as two almost opposite processes, but their actual separation would bring to the disruption of the organism as such: Accommodation alone would bring the organism back to a prebiotic physical condition (full integration into the environment). Assimilation alone would make the organism insensitive to any external stimulus with the consequent lost of plasticity and full annihilation as a consequence of some environmental change.

In the introduction, I have asked whether we are living a paradigm shift. My guess is that we are. The present proposal is of course not meant to achieve such a paradigm shift but simply to do a step forward. The proposed mechanisms - teleonomy and teleology - should indeed be regarded both as innovative explanatory methodology and as fundamental processes actually occurring in the biological domain. As explanatory methods, they do not substitute the molecular and reductionistic approach but point to the possibility, and even the opportunity, to understand biological systems in a non-strictly mechanical way (to whose framework those molecular processes can also fit very well). Should my proposal turn out to be well grounded, a considerable time would be required to fully achieve such a paradigm shift, as far more than mere "cosmetic" work would be needed. The future will tell us how much fruitful the proposed mechanisms will be.

\section{Acknowledgements}

I thank very much Peter R. Wills for his useful comments and suggestions.

\section{References and Notes}

1. Lander, E.S.; Linton, L.M.; Birren, B.; Nusbaum, C.; Zody, M.C.; Baldwin, J.; Devon, K.; Dewar, K.; Doyle, M.; FitzHugh, W.; et al. Initial sequencing and analysis of the human genome. Nature 2001, 409, 860-921.

2. Wilkins, A.S. The Evolution of Developmental Pathways; Sinauer Associates: Sunderland, MA, USA, 2002. 
3. Changeux, J.-P. The Physiology of Truth: Neuroscience and Human Knowledge; Harvard University Press: Cambridge, MA, USA, 2002.

4. Wolpert, L.; Beddington, R.; Jessell, T.; Lawrence, P.; Meyerowitz, E.; Smith, J. Principles of Development; Oxford University Press: New York, NY, USA, 2005.

5. Gilbert, S.F. Devolopmental Biology, 8th ed.; Sinauer Associates Inc.: Sunderland, MA, USA, 2006.

6. Alberts, B.; Johnson, A.; Lewis, J.; Raff, M.; Roberts, K.; Walter, P. The Molecular Biology of the Cell, 5th ed.; Garland Science: New York, NY, USA, 2008.

7. Wolberger, C.; Vershon, A.K.; Liu, B.; Johnson, A.D.; Pabo, C.O. Crystal structure of a MATa2 Homeodomain-Operator complex suggests a general model for Homeodomain-DNA interactions. Cell 1991, 67, 517-528.

8. Bizzarri, M.; Cucina, A.; Conti, F.; D’Anselmi, F. Beyond the oncogene paradigm: Understanding complexity in cancerogenesis. Acta Biotheoretica 2008, 56, 173-196.

9. Kentros, C.G.; Agnihotri, N.T.; Streater, S.; Hawkins, R.D.; Kandel, E.R. Increased attention to spatial context increases both place field stability and spatial memory. Neuron 2004, 42, 283-295.

10. Kuhn, T.S. The Structure of Scientific Revolutions; University Of Chicago Press: Chicago, IL, USA, 1962.

11. Pattee, H.H. Evolving Self-reference: Matter, Symbols, and Semantic Closure. Commun. Cognition-Artif. Intell. 1995, 12, 9-28.

12. Pattee, H.H. The physics of symbols and the evolution of semiotic controls. In The Physics of Symbols and The Evolution of Semiotic Controls; Addison-Wesley: Redwood City, CA, USA, 1997.

13. Hirao, I.; Ohtsuki, T.; Fujiwara, T.; Mitsui, T.; Yokogawa, T.; Okuni, T.; Nakayama, H.; Takio, K.; Yabuki, T.; Kigawa, T.; Kodama, K.; Yokogawa, T.; Nishikawa, K.; Yokoyama, S. An unnatural base pair for incorporating amino acids analogs to proteins. Nat. Biotechnol. 2002, 20, 177-82.

14. Griffiths, P.E. Genetic information: A metaphor in search of a theory. Phil. Sci. 2001, 68, 394-412.

15. Polanyi, M. Lifes irreducible structure. Science 1968, 160, 1308-312.

16. Lehmann, J. Physico-chemical constraints connected with the coding properties of the genetic system. J. Theor. Biol. 2002, 202, 129-44.

17. Holland, S.K.; Blake, C.C.F. Proteins, exons, and molecular evolution. Biosystems 1987, 20, 181-206.

18. Gibbs, W.W. The unseen genome: Gems among the junk. Sci. Am. 2003, 11, 48-53.

19. Shannon, C.E. A mathematical theory of communication. Bell Syst. Tech. J. 1949, 27, 379-423, 623-56.

20. Nielsen, M.A.; Chuang, I.L. Quantum Computation and Quantum Information; Cambridge University Press: Cambridge, UK, 2000.

21. Auletta, G. Quantum information as a general paradigm. Found. Phys. 2005, 35, 787-815.

22. Kauffman, S.A. The Origins of Order; Oxford University Press: New York, NY, USA, 1993.

23. Kauffman, S. At Home in the Universe: The Search for the Laws of Self-Organization and Complexity; Oxford University Press: Oxford, UK, 1995.

24. Ott, E. Chaos in Dynamical Systems; Cambridge University Press: Cambridge, UK, 1993. 
25. Monod, J. Le hasard et la nécessité; Seuil: Paris, France, 1970.

26. Barbieri, M. The Organic Codes: An Introduction to Semantic Biology; Cambridge University Press: Cambridge, UK, 2003.

27. Arthur, W. Mechanisms of Morphological Evolution; John Wiley: New York, NY, USA, 1984.

28. Brooks, C.L. III; Gruebele, M.; Onuchic, J.N.; Wolynes, P.G. Chemical physics of protein folding. PNAS 1998, 95, 11037-38.

29. Russell, E.S. The Interpretation of Development and Heredity; Clarendon Press: Oxford, UK, 1930.

30. Robert, J.S. Embryology, Epigenesis, and Evolution: Taking Development Seriously; Cambridge University Press: Cambridge, UK, 2004.

31. Lotka, A.J. Elements of Physical Biology; Williams and Wilkins: Baltimore, MA, USA, 1925; rep. under the title Elements of Mathematical Biology; Dover: New York, NY, USA, 1956.

32. Parisi, G. Complex systems: A physicist's viewpoint. Physica 1999, A263, 557-64.

33. Woodger, J.H. Biological Principles: A Critical Study? Kegan Paul: London, UK, 1929; Routledge and Kegan Paul: London, UK, 1967.

34. Rosenblueth, A.; Wiener, N.; Bigelow, J.H. Behavior, purpose, and teleology. Philos. Sci. 1943, $10,18-24$.

35. Bichat, F.X. Recherches physiologiques sur la vie et la mort; Brosson: Paris, France, 1800.

36. Gilbert, S.F.; Epel, D. Ecological developmental biology: Integrating epigenetics, medicine, and evolution; Sinauer: Sunderland, MA, USA, 2009.

37. Gould, S.J.; Vrba, E.S. Exaptation: A missing term in the science of form. Paleobiology 1982, 8, 4-15.

38. Blobel, G. Intracellular protein topogenesis. PNAS 1980, 77, 1496-500.

39. Monod, J.; Changeux, J.-P.; Jacob, F. Allosteric proteins and cellular control systems. J. Mol. Biol. 1963, 6, 306-329.

40. Monod J.; Wyman J.; Changeux J.-P. On the nature of allosteric transitions: A plausible model. $J$. Mol. Biol. 1965, 12, 88-118.

41. Hunter, Tony signaling-2000 and beyond. Cell 2000, 100, 113-27.

42. Ma, L.; Cantley, L.C.; Janmey, P.A.; Kirschner, M.W. Corequirement of specific phosphoinositides and small GTP-Binding protein Cdc42 in inducing actin assembly in Xenopus egg extracts. J. Cell Biol. 1998, 140, 1125-136.

43. Martin, T.F. Phosphoinositide lipids as signaling molecules: Common themes for signal transduction, cytoskeletal regulation, and membrane trafficking. Annu. Rev. Cell Dev. Bi. 1998, 14, 231-64.

44. Dippold, H.C.; Ng, M.M.; Farber-Katz, S.E.; Lee, S.-K.; Kerr, M.L.; Peterman, M.C.; Sim, R.; Wiharto, P.A.; Galbraith, K.A.; Madhavarapu, S.; Fuchs, G.J.; Meerloo, T.; Farquhar, M.G.; Zhou, H.; Field, S.J. GOLPH3 bridges phosphatidylinositol-4- phosphate and actomyosin to stretch and shape the Golgi to promote budding. Cell 2009, 139, 337-51.

45. Friston, K.J. A theory of Cortical Responses. Philos. Trans. R. Soc. Lond. B. Biol. Sci. 2005, B360, 815-836. 
46. Friston, K.; Kiebel, S. Predictive coding under the free-Energy principle. Phil. Trans. R. Soc. B 2009, B364, 1211-21.

47. Friston, K.J.; Stephan, K.E. Free-Energy and the brain. Synthese 2007, 159, 417-58.

48. Friston, K.J.; Kilner, J.; Harrison, L. A free energy principle for the brain. J. Physiol. Paris. 2006, 100, 70-87.

49. McKay, D.J.C. A free-Energy minimization algorithm for decoding and cryptanalysis. Electron. Lett. 1995, 31, 445-47.

50. Friston, K.J.; Daunizeau, J.; Kilner, J.; Kiebel, S.J. Action and behavior: A free-energy formulation. Biol. Cybern. 2010, 102, 227-260.

51. Ayala, F.J. Teleological explanations in evolutionary biology. Phil. Scie. 1970, 37, 1-15.

52. Ayala, F.J. Teleological explanations versus teleology. Hist. Phil. Life Sci. 1998, 20, 41-50.

53. Oparin, A.I. The Origin of Life on the Earth; Academic Press: New York, NY, USA, 1957.

54. van Gelder, T. Compositionality: A connectionist variation on a classical theme. Cognitive Sci. 1990, 14, 355-84.

55. Berg, J.M.; Tymoczko, J.L.; Stryer, L. Biochemistry, 6th ed.; Freeman and Co.: New York, NY, USA, 2006.

56. Crick, F.H.C. The central dogma of molecular biology. Nature 1970, 227, 561-63.

57. Jeong, H.; Tombor, B.; Albert, R.; Oltvai, Z.N.; Barabási, A.-L. The large-scale organization of metabolic networks. Nature 2000, 407, 651-654.

58. Jeong, H.; Mason, S.P.; Barabási, A.-L.; Oltvai, Z.N. Lethality and centrality in protein networks. Nature 2001, 411, 41-42.

59. Wagner, A. Robustness and Evolvability in Living Systems; Princeton University Press: Princeton, NJ, USA, 2005.

60. Auletta, G.; Ellis, G.; Jaeger, L. Top-down causation by information control: From a philosophical problem to a scientific research program. J. R. Soc. Interface 2008, 5, 1159-1172.

61. Wegscheid, B.; Condon, C.; Hartmann, R.K. Type A and B RNase P RNAs are interchangeable in vivo despite substantial biophysical differences. EMBO Reports 2006, 7, 411-17.

62. Gobert, A.; Gutmann, B.; Taschner, A.; Gössringer, M.; Holzmann, J.; Hartmann, R.K.; Rossmanith, W.; Giegé, P. A single Arabidopsis organellar protein has RNase P activity. Nat. Struct. Mol. Biol. 2010, 17, 740-46.

63. Weiner, O.D. Regulation of cell polarity during eukaryotic chemotaxis: The chemotactic compass. Curr. Opin. Cell Biol. 2002, 14, 196-202.

64. Alon, U. Simplicity in biology. Nature 2007, 446, 497.

65. Jurica, M.S.; Stoddard, B.L. Mind your Bs and Rs: Bacterial chemotaxis, signal transduction and protein recognition. Structure 1998, 6, 809-813.

66. Parkinson, J.S. Signal transduction schemes of bacteria. Cell 1993, 73, 857-71.

67. Rao, C.V.; Kirby, J.R.; Arkin, A.P. Design and diversity in bacterial chemotaxis: A comparative study in Escherichia coli and Bacillus subtilis. PLOS Biol. 2004, 2, 0239-52.

68. Minelli, A. The Development of Animal Form: Ontogeny, Morphology, and Evolution; Cambridge University Press: Cambridge, UK, 2003.

69. Buss, L.W. The Evolution of Individuality; Princeton University Press: Princeton, NJ, USA, 1987. 
70. Arthur, W. The Origin of Animal Body Plans: A Study in Evolutionary Developmental Biology; Cambridge University Press: Cambridge, UK, 1997.

71. Schlichting, C.D.; Pigliucci, M. Phenotypic Evolution: A Reaction Norm Perspective; Sinauer: Sunderland, MA, USA, 1998.

72. Wright, S. Evolution in mendelian populations. Genetics 1931, 16, 97-159.

73. Wright, S. The roles of mutation, interbreeding, crossbreeding and selection in evolution. In Proceedings of the Sixth International Congress on Genetics, Ithaca, New York, NY, USA, 1932; pp. 56-66.

(c) 2010 by the author; licensee MDPI, Basel, Switzerland. This article is an open access article distributed under the terms and conditions of the Creative Commons Attribution license (http://creativecommons.org/licenses/by/3.0/.) 